\title{
21. DISCUSSION FOLLOWING THE REPORTS BY POTTASCH AND BOYARCHUK
}

(Sunday, September 14, 1969 and Tuesday, September 16, 1969)

\author{
Chairmen: R. WeYMANN and S. A. KAPLAN
}

\begin{abstract}
Editor's remark: This Discussion contains the remarks made during the discussion after Boyarchuk's Report on Tuesday, September 16, and about half of the remarks made in the discussion after the Reports on Sunday, September 14. The other half of the latter discussion is presented in Chapter 18. At the end of the Session on Tuesday Thomas asked Busemann to comment on the interrelation between aerodynamics and interstellar gas dynamics. Busemann's response has been transferred to the Final Discussion (Chapter 27). Some general comments by Goldsworthy have been moved to Chapter 5, Section 6.
\end{abstract}

\section{Dynamical Interaction of Stellar Winds and Surrounding Matter}

Weymann: Inasmuch as we are dealing with aspects of the interstellar medium, Pikel'ner would like to try to tie today's deliberations into this general topic.

Pikel'ner: I would like to discuss the interaction of stellar winds with matter in diffuse nebulae. Shcheglov at the Sternberg Institute in Moscow has observed radial motions of little blobs of gas in the Omega Nebula and some other nebulae. The velocities of these motions go up to 50 to $70 \mathrm{~km} \mathrm{sec}^{-1}$. At the Prague meeting of the IAU Menon suggested that such velocities may be connected with stellar winds blowing from stars inside the Nebula. I have made calculations for such a process (Pikel'ner and Shcheglov, 1968) which I want to discuss now. Suppose that we have a star, which produces a stellar wind, and a nebula around it. Usually one makes the assumption that the wind produces a thin shell of compressed interstellar gas having a radius $R$ from the star and moving away at a velocity $V$. One derives easily from momentumconservation and energy-conservation equations (see Pikel'ner and Shcheglov, 1968) that $V R=$ constant. The constant depends on a number of known parameters and can be estimated. If this model is applied to the Omega Nebula, one obtains $V \approx 50$ to $100 \mathrm{~km} \mathrm{sec}^{-1}$ at $R \approx 0.2 \mathrm{pc}$; the observed value of $R$ is, however, 1 to $2 \mathrm{pc}$. I therefore considered a different model, which is shown in Figure 1. In addition to the layer of compressed interstellar gas (layer 3 in Figure 1) we have a layer of compressed wind (layer 2), that is not very thin, as was assumed previously. I assumed a stellar wind velocity of $1500 \mathrm{~km} \mathrm{sec}^{-1}$. Since in shock front $I$ the kinetic energy of the stellar wind is converted almost completely into thermal energy, the temperature in layer 2 is about $10^{8} \mathrm{~K}$. The density in layer $2, n_{2}$, is about $0.1 \mathrm{~cm}^{-3}$, so the gas cools very slowly by radiation and adiabatic cooling dominates. Since the sound speed in layer 2 is very large, the pressure $p_{2}$ will be uniform. It is easily shown that $p_{2} \propto R_{1}^{-2}$. It is also seen that 


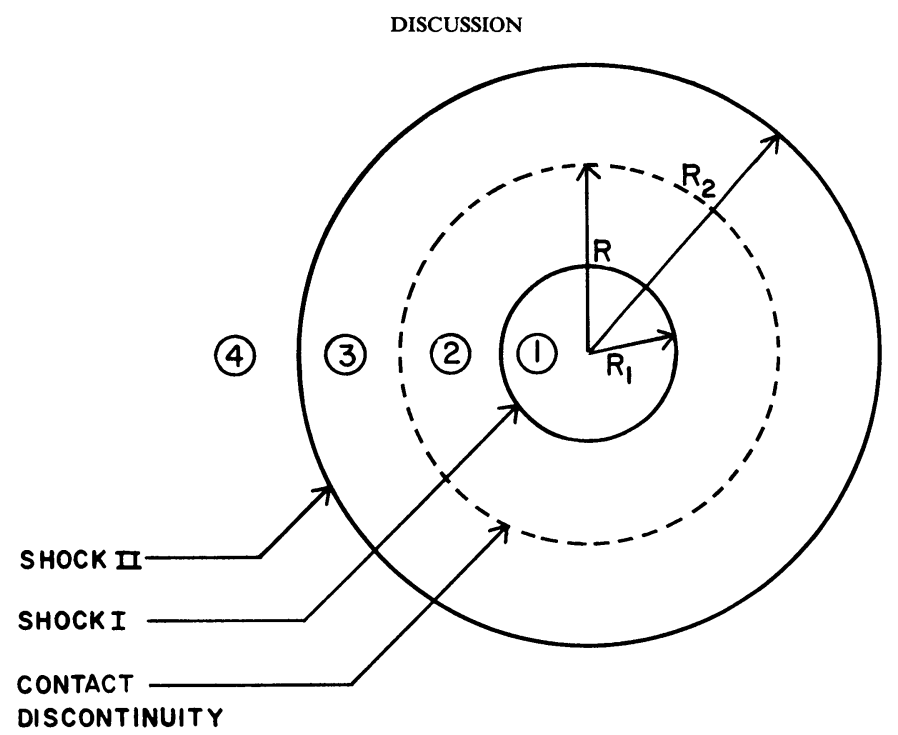

Fig. 1. (See the remark by Pikel'ner.) Schematic picture of the different zones connected with outflow of matter from a star, which is put at the center. 1 and 2 are the stellar wind region; 3 and 4 are the interstellar gas region.

shock front II is driven by $p_{2}$. For if the velocity $v_{\text {II }}$ of shock front II were too low, $R_{1}$ would decrease and $p_{2}$ would increase, which would increase $v_{\mathrm{II}}$. This situation resembles a hydraulic press. Calculations show that layer 3 is very compressed $\left(n_{3}=10^{3} \mathrm{~cm}^{-3}\right.$; layer thickness $10^{-2} \mathrm{pc}$ ). It is the layer that shows up in the observations; it can have a velocity of 50 to $70 \mathrm{~km} \mathrm{sec}^{-1}$, even for $R \approx R_{2} \approx 1$ to $2 \mathrm{pc}$. The emission measure of layer 3 is about $10^{4} \mathrm{~cm}^{-6} \mathrm{pc}$, which is large enough to explain the observations. The velocity of this layer is determined essentially by the equation $p_{2} \approx \varrho_{4} v_{\mathrm{II}}^{2}$, its temperature by the UV flux from the central star. If the density in layer 4 is not uniform, shock front II will not maintain its spherical symmetrical shape. Let us suppose that there are some globules in layer 4 . The globule will not share in the motion and, after some time, it will be in layer 1, where the surrounding pressure is ten or more times higher than in layer 4 . The globule will therefore be compressed to a density of perhaps $10^{5} \mathrm{~cm}^{-3}$. Fluctuations in the interstellar gas density can increase in the same way. Perhaps the high emission measure of the center of the Orion Nebula may be explained by a compression of the kind described.

Finally I tried to apply these ideas to Wolf-Rayet stars. There are several WolfRayet (WR) stars with a nebula around them; the best known of such nebulae is NGC 6888, which has a filamentary structure. This nebula, together with several others, has been studied recently by T. Losinskaia. Together with V. Avedisova and T. Losinskaia I made calculations to explain these nebulae as a consequence of stellar winds coming from the WR stars. We took a mass loss of $10^{-5} M_{\odot}$ per year and a wind velocity of $1400 \mathrm{~km} \mathrm{sec}^{-1}$. Calculations give an expansion velocity for the nebulae up to $100 \mathrm{~km} \mathrm{sec}^{-1}$, which is in agreement with observations. The elongated form of the nebulae cannot be explained by the influence of magnetic fields unless the field strength 
is at least $100 \mu \mathrm{G}$. So it seems more reasonable to suppose the stellar wind to be asymmetric. [Pikel'ner, S. B. and Shcheglov, P. V.: 1968, Astron. Zh. 45, 953 (translation: 1969, Soviet Astron. 12, 757).]

Thomas: Why would there be an asymmetry of the stellar wind?

Pikel'ner: I do not know. Perhaps active surface regions give a stronger wind than quiet regions, as in the Sun.

Dyson: Two comments. First, Dr. Meaburn of the University of Manchester has made observations with a Fabry-Pérot interferometer on several nebulae observed by Shcheglov and has not confirmed the 50 to $70 \mathrm{~km} \mathrm{sec}^{-1}$ velocities. Second, I have a comment on the suggestion that ionized globules are compressed by the expanding stellar wind. In the case of the Orion Nebula, there is some disagreement as to where the Trapezium stars should be located relative to the ionized gas. There is a possibility that the Trapezium is not spatially associated with the larger part of the ionized gas comprising the Nebula. The observations of Münch and Wilson seem to indicate splitting of lines, probably due to shock waves, associated with the densest regions of the Nebula. Therefore it is possible that the displacement of the exciting stars may cause this mechanism to be ineffective.

Pikel'ner: Shcheglov thinks that his very sensitive photoelectric equipment permits the use of his Fabry-Pérot étalons with higher accuracy. I cannot discuss Meaburn's observations since I am not an observer. I try only to explain observations made by others. But, in any case, if stars have winds of the indicated magnitude the winds affect the surrounding nebulae in about the way I indicated. As for the Orion Nebula, Menon pointed out, several years ago, the difficulty that the very steep gradient of surface brightness in the center of the Nebula indicates a density concentration which will expand in a few thousand years. This difficulty has not been solved before, so far as I know. It has been suggested (see e.g., the Report by Mezger, p. 336) that there are strong density fluctuations in the center of the Orion Nebula, since locally high emission measures are observed. Such fluctuations can persist for a considerable time only if there is some external pressure which keeps them in quasi-equilibrium. So if the model I described is not valid, it is necessary to find some other explanation for the high external pressure.

Dyson: Is there a possibility of confusion with the forbidden N II lines?

Pikel'ner: I do not think so, that mistake seems too elementary to make.

Dyson: I have proposed a somewhat different mechanism involving the existence of neutral condensations in the central regions of the Orion Nebula. If the ionized blobs in the Orion Nebula are simply fluctuations in the ionized gas, they will smooth out rapidly due to expansion, as Pikel'ner has mentioned. Consider however, the situation where the density fluctuation is of neutral hydrogen. On the surface of this condensation is an ionization front which eats its way into the neutral gas. Ionized gas streams off the surface with a supersonic velocity. The interaction of these supersonic gas streams produces a system of shock waves. Computed line profiles for the simplest case of interaction of two streams from adjacent condensations are in good agreement with the results given by Münch and Wilson. Admittedly one should take into account 
other emission in the line-of-sight which would affect the detailed profiles. This mechanism would not produce velocities much greater than perhaps 30 to $40 \mathrm{~km} \mathrm{sec}^{-1}$.

Thomas: These problems were also discussed by Lindsey Smith in the Symposium on Wolf-Rayet stars held in Boulder, Colorado, in 1968 (Gebbie and Thomas, 1968). Expanding shells carrying material out from WR stars were discussed classically a long time before the concept of a stellar wind was introduced. A stellar wind implies the existence of a high temperature corona as discussed first by Parker. The old idea was simply one of an initial expansion, without any idea of the source of the expansion; and no one thought of the WR star as having a corona. Lindsey Smith posed the problem of why only a few, and not all WR stars have such nebulae. If I remember correctly, she concluded that an observable nebula could only be swept up in regions of space having a higher space density than normal. In other regions of space, there is too little material. (Gebbie, K. B. and Thomas, R. N. (eds.): 1968, Wolf-Rayet Stars, U.S. Government Printing Office, Washington, D.C.)

Stecher: In this connection, Bless, Fischel, and I suggested we might get X-ray emission from the expanding shells, when they run into the interstellar medium. We also used a figure of $10^{-5} M_{\odot} \mathrm{yr}^{-1}$ for the mass loss. We tentatively suggested as a candidate the WR star, $\gamma$ Velorum, which is now observed to be emitting low-energy $\mathrm{X}$-rays quite strongly. So, Pikel'ner's model seems quite reasonable to me.

Silk: If I remember well, in that area of the sky there is quite a large margin of error as to which of the objects actually emits X-rays.

Stecher: Yes, the observations have only about $1^{\circ}$ resolution.

Pottasch: Dr. Pikel'ner, a question on your mass loss of $10^{-5} M_{\odot} \mathrm{yr}^{-1}$ for WR stars. Is that a result of your calculations or an assumption?

Pikel'ner: An assumption, taken from what is in the literature.

Pottasch: Then I caution against the use of this figure. It's derived by assuming the mass-loss proportional to $R^{2} V \varrho$, and the density values at the point where we know the velocity are so uncertain that a great deal of reserve is necessary when using this number.

Pikel'ner: I agree. But we relied also on Morton's statement that the usual OB stars have a mass loss of some $10^{-6} M_{\odot} \mathrm{yr}^{-1}$. The envelopes of WR stars are brighter and therefore should have a higher mass loss.

Thomas: I went through these arguments before (Thomas, 1949), using the data on the density distributions in 444 Cygni as determined by Shapley and Kopal. I reached a figure of $10^{-5} M_{\odot} \mathrm{yr}^{-1}$ by using the observed velocity. I pointed out that this was a very dubious result, because, for example, $\gamma$ Velorum has not changed its magnitude in about 3000 years; and with such a high rate of mass loss the star would lose several per cent of its mass over this period. If you find such a high mass loss rate, you must argue that a several per cent change in the mass does not appreciably affect the luminosity. I therefore agree completely with Pottasch's caution. (Thomas, R. N.: 1949, Astrophys. J. 109, 500.)

Weymann: The photometric errors were quite large 3000 years ago.

Thomas: That does not matter. Relative photometry was fairly good; and in 
3000 years $\gamma$ Velorum has not changed its relative magnitude by more than a half magnitude. How much change in luminosity do you expect from a few per cent mass loss?

Pikel'ner: Perhaps the WR state is a very short one in the life of a star. If a star loses a significant portion of its mass, its luminosity should change. But I am not discussing these stars; I am using published figures to compute the effects on the interstellar medium.

Mestel: There exists an upper limit on the mass loss rate derived by Lucy and Solomon (1970). Their argument does not deal with the classical stellar wind model, but with ejection under radiation pressure in resonance lines. The upper limit is just $L / c^{2}$ where $L$ is the stellar luminosity. This means that the mass loss is never greater than the mass loss from conversion of hydrogen into helium. (Lucy, L. B. and Solomon, P. M.: 1970, Astrophys. J. 159, 879.)

Field: Can one convert that upper limit to solar masses per year? [For a star with $M_{\text {bol }}=-8$ one finds $L / c^{2}=7 \times 10^{-9} M_{\odot} \mathrm{yr}^{-1}$. According to Table I in Pottasch's Report, p. 273, Morton gives a mass loss rate of $1.5 \times 10^{-6} M_{\odot} \mathrm{yr}^{-1}$ for OB stars. (Ed.)]

Silk: Jokipii (1969) has suggested that the low-energy cosmic rays between 1 and 2 $\mathrm{MeV}$, that have been observed near the Earth, have been produced in the region of interaction between the solar wind and the interstellar gas. My question is a more general one. Could someone comment on the general contribution of stellar winds and their interactions to an interstellar low-energy cosmic-ray flux? (Jokipii, J. R.: 1968, Astrophys. J. 152, 799.)

Parker: The contribution to interstellar space would be extremely small. The suggestion by Jokipii is that the interaction of the wind with the interstellar medium would generate a high density of low-energy cosmic rays, within the region occupied by the solar wind. That is a small region. I do not think that it would significantly affect the abundance of the low-energy cosmic rays in interstellar space. It is too small a contribution to fill that vast a region.

Silk: Would this also apply to the OB stars possessing more energetic stellar winds?

Parker: I would have to do some arithmetic before I can answer.

\section{Evidence of Stellar Winds}

Stecher: I would like to show two slides with direct observations of stellar winds from OB stars (Figures 2 and 3). Figure 2 shows the UV spectrum of the star $\zeta$ Puppis, an O5 star, that - with $\gamma$ Velorum - illuminates the Gum Nebula. The wavelength range is from 1000 to $2500 \AA$. Note the CIV lines around $1500 \AA$ both in emission and in absorption within a $100 \AA$ interval. The separation of the peaks corresponds to $15 \AA$, or $3000 \mathrm{~km} \mathrm{sec}^{-1}$. The same combination of emission and absorption in one line shows up in all the resonance lines of the star. One would assume then a model, in which the velocity is low close to the star, but increases as one goes out because of the density decrease. The emission is produced by a shell around the star. The absorption 


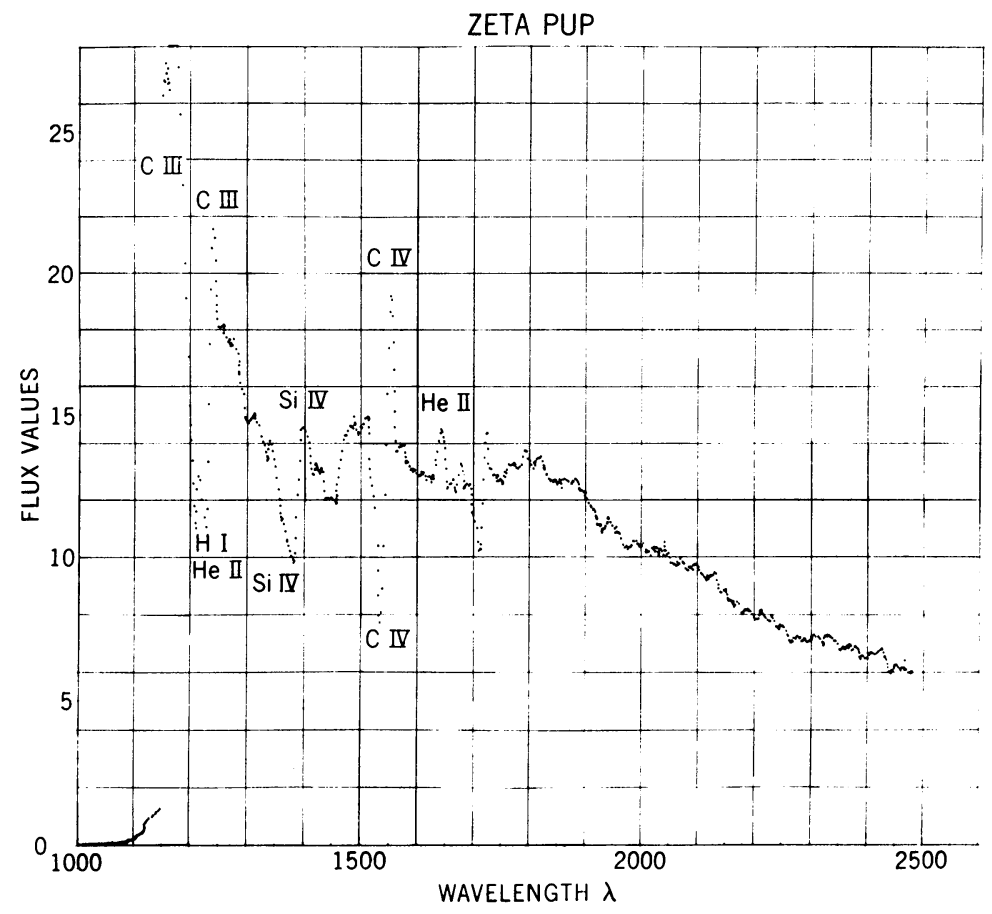

Fig. 2. (See the remark by Stecher.) The spectrum of $\zeta$ Pup between 1000 and $2500 \AA$. The flux is in units of $10^{9} \mathrm{erg} \mathrm{cm}^{-2} \mathrm{sec}^{-1} \AA^{-1}$ at the top of the Earth's atmosphere.

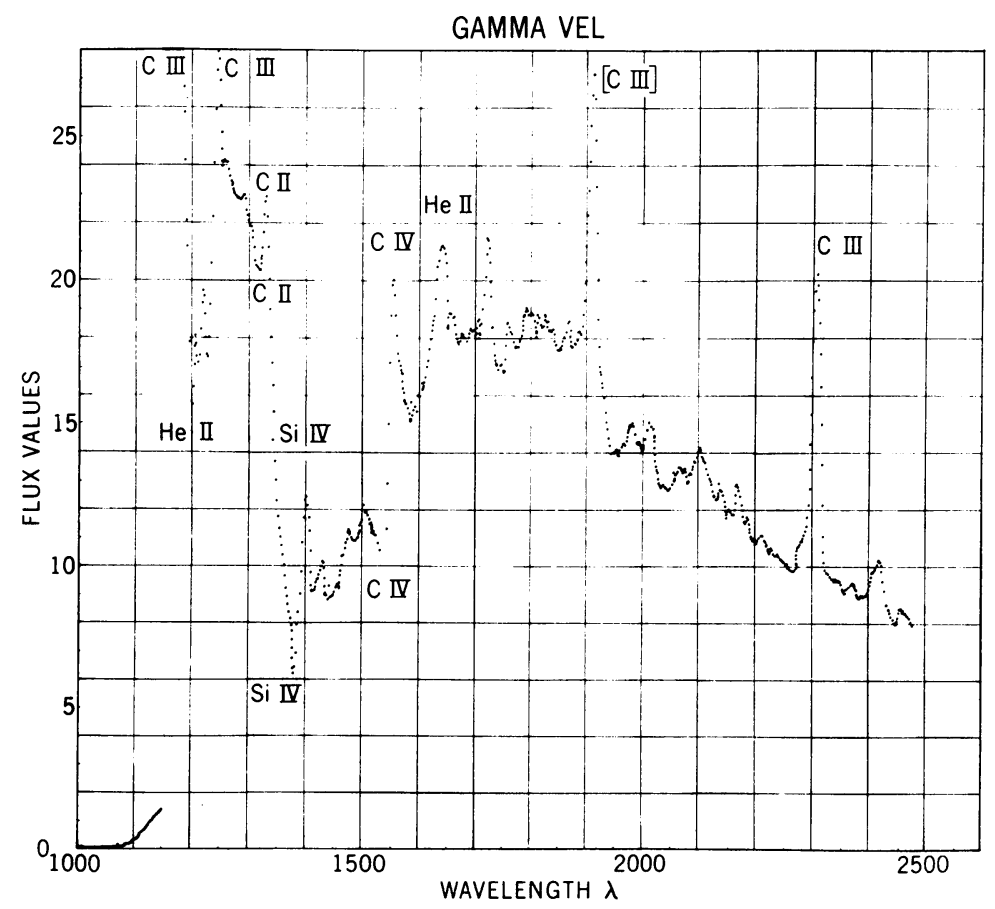

Fig. 3. (See the remark by Stecher.) The spectrum of $\gamma$ Vel between 1000 and $2500 \AA$. The flux is in units of $10^{9} \mathrm{erg} \mathrm{cm}^{-2} \mathrm{sec}^{-1} \AA^{-1}$ at the top of the Earth's atmosphere. 
line originates in shell matter moving away from the star and seen in projection against the stellar disk. The inferred velocity of the gas is model-dependent because some of the absorption may occur against the emitting shell, instead of against the stellar disk. The total amount of gas flowing out is model-dependent because we do not know whether the line broadening is radiation damping or Doppler broadening. Figures 2 and 3 contain photoelectric observations obtained with a $32-\mathrm{cm}$ telescope, flown in a rocket. Figure 3 shows a WR star, $\gamma$ Velorum which, as I mentioned, also participates in the excitation of the Gum Nebula. Again CIV shows in emission and absorption with about half the Doppler shift of $\zeta$ Puppis, but the density is higher. The line at $\lambda 1909$ is an intercombination line of CIII with an $A$-value of $190 \mathrm{sec}^{-1}$, while the $\lambda 2296$ line is a permitted line in the singlets of Cill with an $A$-value of $10^{8} \mathrm{sec}^{-1}$. D. K. West and I are now engaged in producing a set of models and attempting to derive density and mass loss from this star. We have no final conclusions yet.

Thomas: I would remark on the reliability of a direct, naive interpretation of such results as Stecher summarized; his statement that the results are model-dependent should be in flashing letters. Stecher's interpretation is the historical one, applied originally to WR and P Cyg spectra. It assumed a classical model atmosphere, with excitation and electron temperature decreasing outward, surrounded by an optically thin, tenuous atmosphere expanding outward. Possibly there are dilution effects; but aside from these all excitation functions are derived from LTE. In this model the emission lines are observed only because the line-producing region has a larger volume than the continuum-producing region. But this kind of model is probably wrong. I argued many years ago that the WR atmosphere was one big chromosphere-corona and that the interpretation of the line-profiles must be based on the same kind of physics we use for the solar outer atmosphere. The evidence is now very strong that this suggestion was correct; you may consult the Proceedings of the Wolf-Rayet Symposium (Gebbie and Thomas, 1968). Recent work by Castor at the Joint Institute for Laboratory Astrophysics in Boulder gives strong evidence for optical depth effects; hence radiative transfer must be included. All of these considerations must be applied to interpret these new rocket UV spectra of the supergiants. On this basis, please note that the observation of the emission line profiles gives us the possibility of inferring density distribution, electron temperature distribution, and velocity gradients. The simplest illustrations of the techniques arise in the Sun, where the velocities and the velocity gradients are not so high, but where the effects are present. These are the reasons we return always to the Sun as a guide - in the solar-stellar wind, in the solarstellar line profiles. So, be cautious, and demand physical consistency of models. (Gebbie, K. B. and Thomas, R. N. op. cit.)

Field: Dr. Thomas, is there any escape from the qualitative conclusion that there is an outflow of about $1000 \mathrm{~km} \mathrm{sec}^{-1}$ from the star, even though the amount of such flow is very uncertain?

Thomas: No, there is no uncertainty that such high velocities exist. The uncertainty lies in the exact value of the outflow density, and in where it occurs in the atmosphere. 
One wants to base a final interpretation on a completely realistic model of the atmosphere, viewing it as one in which the electron temperature increases outward, due to the mechanical heating, which in turn arises from the aerodynamical motions whose geometry we try to infer. Let me remind you that, before these observations, most astronomers disbelieved that such stars even had chromospheres-coronas and the required underlying aerodynamic motions. The preobservational predictions were based wholly on the classical, non-aerodynamical, non-chromospheric models. Let us please be physically consistent, now, in making any more models and interpretations.

Stecher: To amplify Thomas's remark: In one case we have tried to compute a model. Now take the intercombination line of CIII, at $\lambda 1909$. It is barely thick in the thermal case. That is, by solving the transfer problem in the moving atmosphere we can reproduce the equivalent width of the emission line. The line has no absorption core and we have an opacity due to electron scattering of about $10^{-2}$. For the resonance line $\lambda 977$, from which we are trying to get the mass-flow, this model implies a many-times-greater opacity in electron scattering. I agree completely with Thomas that the problem is very difficult.

Field: Would Dr. Pottasch comment on his statement that some stars having observable mass outflow in the UV had not been observed to show this in the visual. What about the reverse? And is there any star where we observe mass loss in both spectral regions?

Pottasch: In the three stars observed by Morton, which I discussed, there is essentially little or no evidence for mass loss in the visible part of the spectrum (no P Cygtype profiles). In one star you do not see anything at all which you could associate with mass loss. In the other two stars, you do see $\mathrm{H} \alpha$ in emission. But before you saw the UV spectra nothing would have led you to suppose that there was a great deal of mass loss.

Weymann: In one of these objects Abt looked very hard for the metastable He line at $3389 \AA$. There was no sign of it.

Field: Are there many stars where you do see evidence for mass outflow in the visible spectrum? Are they the same type of stars?

Pottasch: They are exactly the same type of stars. Perhaps the difference is that they are somewhat more luminous. If you were to make a semi-qualitative plot of evidence of mass loss - say, P Cyg profiles, helium absorption, and $\mathrm{H} \alpha$ emission - there would be a correlation between the luminosity of the star and these features.

Field: What about the stars Stecher described?

Pottasch: Both have been observed in the visible. One is a WR star. There, the evidence for mass loss is the conventional one inferred from broad profiles. It is hard to see how you can avoid some sort of mass loss from WR stars.

Stecher: The star $\zeta$ Puppis has emission lines in the visible, and thus some indication of mass loss. Ten years ago, Robert Wilson in Edinburgh found some absorption lines that were blue-shifted, suggesting mass loss. But the lines were very weak.

Bisnovatyi-Kogan: There has been some discussion on the effects of mass loss on the evolution of a star. I want to mention some computer results on mass loss of massive 
stars during their evolution. Madejin and I are computing the evolution of stars with large masses, not just the static phase, but also in the phase when there is a static core and outflow of matter in the atmosphere. The important point is that the rate of mass loss is not arbitrary, but follows from the theory and is a unique function of the stage of evolution. The preliminary results for $30 M_{\odot}$ stars are as follows: after the production of a helium core and the beginning of helium burning the surface temperature reaches a value of about $4600 \mathrm{~K}$, the luminosity about $2.3 \times 10^{5} L_{\odot}$, and the radius about $610 R_{\odot}$. At this moment the star begins to lose mass rapidly. The time of mass loss is smaller than the time of nuclear burning. We expect that mass will continue to flow out until the star has lost about $15 M_{\odot}$ and the hydrogen layer source will be exhausted. The computation for a $9 M_{\odot}$ star did not give a large mass loss during the phase of helium burning.

Weymann: Can you describe briefly the mechanism responsible for the mass outflow?

Bisnovatyi-Kogan: The physical mechanism is radiation pressure. The mechanism works because of high opacity in the zone of incomplete hydrogen ionization. [Bisnovatyi-Kogan, G. S. and Zel'dovich, Ya. B.: 1968, Astron. Zh. 45, 241 (translation: 1968, Soviet Astron. 12, 192).]

Stecher: Dr. Boyarchuk, I noticed that in your list the $\mathrm{T}$ Tau stars have a mass loss of $10^{-7} M_{\odot} \mathrm{yr}^{-1}$. That seems more than the upper limit set by Lucy and Solomon on account of radiation pressure. Are there other stars in your list that are not luminous enough to have radiation pressure as a driving mechanism?

Boyarchuk: I do not know about the correct mechanism for the mass loss from $\mathrm{T}$ Tau stars. Many people think that it may be something like prominence activity. But it seems to me that for none of the eruptive stars do we have a good mechanism that can produce mass loss.

Mestel: Is it ruled out that in the $\mathrm{T}$ Tau phase we are seeing essentially a violent Parker-style stellar wind? It should be remembered that the wind theory does not in itself determine the mass loss; the density at the coronal base (or alternatively at the sonic point) is known only when we have a model for the chromospheric-coronal structure for the particular star considered. But it seems to me that the T Tau phenomenon will be more usefully studied as a quasi-stationary rather than as a nonstationary phenomenon.

Thomas: Again I should like to make the point that in discussing mass loss one has to make a distinction. First there is the sort of model that Boyarchuk used; it consists only of an expanding envelope. But second, we have the solar-wind-type models. And there we do not know what produces the corona. There must be some sort of mechanical energy transport going up in the atmosphere. I think we should discuss here the fundamental thing, the production of the chromosphere-corona. If, as some of us have argued, all stars probably have chromosphere-coronae (not just those stars for which there is a convective zone that can become unstable), then this subject is a whole region of interest which we must consider.

Mestel: I am not opposed to this view; however, it is worth remembering that a 
magnetically-controlled centrifugal wind can cause substantial mass loss from a rapidly rotating star, even if the surrounding corona is cool. The mechanism is clear: the magnetic field tries to preserve co-rotation, and the centrifugal forces drive the gas out. The Parker critical point is now given by the Bernoulli equation, modified to include the rotational terms. If the star rotates rapidly, then on most of the fieldstream lines this point is at a level where the density is high. Of course, the process involves loss of angular momentum. If the star is not contracting then its rotation rate slows down, the sonic point moves out to a position of lower density, and the process cuts off. But $\mathrm{T}$ Tau stars are contracting towards the main sequence, and so can maintain a powerful centrifugal wind.

\section{Mass Loss from Binary Systems}

Krat: I want to comment on mass interchange in close binaries. If the time scale of mass interchange is of the order of $10^{5}$ or $10^{6} \mathrm{yr}$, it can be comparable to the time scale of propagation of energy from the inner parts of a star to its boundary. If the mass of a star changes significantly on a time scale less than $10^{5} \mathrm{yr}$ due to a superficial process of mass capturing or to mass ejection, and if the luminosity remains constant, because the changes in internal energy production take longer to reach the boundary, then certain kinds of instabilities will develop. The expanding star will accelerate its expansion, and the star receiving the flow of extra mass will contract. The first process will form rarefied gaseous shells around the expanding star; the second will lead to a burst, and the star can become variable. In some specific cases, supernova and nova explosions can occur. The short time scale of mass interchange changes a continuous evolution process to a disruptive one and radically changes the composition of stellar populations and the chemical composition of interstellar matter. Vice versa the interstellar material can be processed in a comparatively short time. The time scale of the interchange processes plays a very important role in the cosmogenic picture of stellar evolution.

Gorbatskii: I want to comment on the input of matter from close binaries to the interstellar medium. In close binaries there are gaseous streams forming disk-like envelopes and jets. For binary systems of very small absolute luminosity $\left(M_{v} \approx+9^{m}\right)$ such as old novae, U Gem stars and similar ones, the gaseous streams are very important for the explanation of the observed features. Recently gaseous streams in such systems were studied theoretically at the Leningrad University (Gorbatskii, 1967). The motion of gaseous jets was calculated, including expansion of the jet due to gaseous pressure as well as the effects of centrifugal forces and Coriolis forces. For a wide range of initial conditions a fraction of the gas escapes from the system due to gaseous pressure at the first Lagrangian point; the escaping fraction of the jet can be as much as 0.5 . By comparing the theory with the observational data it was found that the gas flow from the jet to the surface of the other star is of the order of $10^{-10} M_{\odot} \mathrm{yr}^{-1}$. The total number of the binaries under discussion in the Galaxy may be about $10^{8}$. Therefore the mass loss from all these systems may be as high as $10^{-2} M_{\odot} \mathrm{yr}^{-1}$. There are 
many more close binaries of other types in the Galaxy that contain gaseous streams and may lose mass effectively. The mass loss from such systems has not been considered quantitatively yet, but many reasons let us believe that the total mass loss from close binaries may amount to $1 M_{\odot} \mathrm{yr}^{-1}$. This is of much importance for the mass balance of the interstellar medium. I have a second comment referring to the heating of interstellar medium by UV and X-ray radiation emitted by close dwarf binaries. Calculations show that the velocity of the jet, when it collides with the disk-like envelope, is of the order of $500 \mathrm{~km} \mathrm{sec}^{-1}$. The collision leads to a strong shock wave which corresponds to what the observers call a 'hot spot'. The gas is heated in the shock to a temperature of several million degrees, and emits mainly in the far UV and soft X-ray region. The total energy radiated in this range for the Galaxy may be $10^{39}$ to $10^{40}$ erg sec${ }^{-1}$. This is a considerable quantity compared with the output of other UV and X-ray sources in the Galaxy. [Gorbatskii, V. G.: 1967, Astrofiz. 3, 245 (translation: 1968, Astrophys. 3, 116).]

Field: Did you skip a decimal? Did you say $1 M_{\odot} \mathrm{yr}^{-1}$ ?

Gorbatskii: Correct, $1 M_{\odot} \mathrm{yr}^{-1}$ from close binaries. Boyarchuk mentioned an even larger figure from U Gem stars alone. Commenting upon Boyarchuk's figure of $1.8 \times 10^{-7} M_{\odot} \mathrm{yr}^{-1}$ for SS Cyg, I would like to say that, if we have a mass loss of that order of magnitude, a very dense envelope will develop around the binary system and will make the system invisible. However, U Gem, SS Cyg and others are very clearly observed with sharply defined lines at the minimum. This fact indicates that the mass loss is at most $10^{-8} M_{\odot} \mathrm{yr}^{-1}$.

Mestel: Since I presented Salpeter's paper, I feel obliged to comment on one point. Boyarchuk gave a very high upper limit of $1 M_{\odot} \mathrm{yr}^{-1}$ for mass loss from $\mathrm{U}$ Gem stars alone, as compared with Salpeter's figure of a fraction of $1 M_{\odot} \mathrm{yr}^{-1}$ from stars of all types. The figures are reached by quite different methods: Boyarchuk's, quasiempirically; Salpeter's, from a general theoretical picture of the evolution of stars into white dwarfs. Salpeter also estimated current star formation at a rate of $1 M_{\odot} \mathrm{yr}^{-1}$. If we accept this star formation figure together with Boyarchuk's upper limit on mass injection in interstellar space, we must conclude that the interstellar medium may actually be gaining mass at the moment, and primarily from members of $U$ Gem systems, which will for the same reason end up as white dwarfs of very low mass. (My own feeling is that future work will greatly reduce the contribution of $U$ Gem stars.)

We should also note that if Kuhi's results on T Tau stars are accepted (see the Report by Boyarchuk, p. 281), then during this pre-main sequence stage, mass loss can sometimes be so great as to affect very markedly stellar evolution computations, as noted in particular by Iben. [See also Kuhi, L. V. and Forbes, J. E. : 1970, Astrophys. J. 159, 871. (Ed).]

Drobishevskii: I should like to show that a classification of magnetic stars supports the evidence for mass ejection by some types of stars. (1) I want to point out two difficulties with the hypothesis that magnetic stars are to be found among those with large convection zones and rapid rotation. The first difficulty is that most magnetic 
stars are of spectral class $\mathrm{A} 1$ and earlier $\left(M_{*} \geqslant 3 M_{\odot}\right)$ where the convective flux is unimportant (the maximum flux is attained between spectral types A5 and F0). The second difficulty is that the magnetic field, generated in the convective envelope, diffuses too slowly into the interior, radiative equilibrium part of the star. Diffusion by ohmic dissipation takes a time equal to or longer than the stellar age. (2) Both difficulties disappear if one postulates that mass accretion on the stars forces the convective zone to move outward, leaving the frozen-in magnetic field behind in the radiative equilibrium zone. One can put lower limits to the mass accretion rate in two ways. One: the accretion rate has to be so large that the rate at which gas flows into the interior of the star is longer than the rate at which magnetic energy diffuses out of the radiative equilibrium zone. Two: the rate of generation of magnetic flux in the convective zone has to be at maximum. Both lower limits turn out to be $10^{-12} M_{\odot}$ $\mathrm{yr}^{-1}$ to $10^{-13} M_{\odot} \mathrm{yr}^{-1}$ for stars of types A through F. (3) It is certain that accretion of matter from interstellar space is smaller than the lower limits presented. Therefore we consider the possibilities of mass-exchange in close binaries under the supposition that essentially there are two types of mass outflow from stars: one, a stellar windtype outflow, to be found in stars with $1 M_{\odot} \leqslant M_{*} \leqslant 1.5 M_{\odot}$. Two, a large outflow of unspecified nature in stars with $M_{*} \geqslant 5 M_{\odot}$. On this supposition we predict the existence of three groups of magnetic stars. Group 1, where the stellar mass $M_{* 1}$ is between 1.5 and $3 M_{\odot}$; group 2 , where $M_{* 2}=M_{* 1}+1.5 M_{\odot}$; and group 3 where $M_{* 3}=M_{* 1}+5 M_{\odot}$ (4) The prediction of the existence of three groups of magnetic stars is in agreement with the results obtained by Babcock, Preston, and others. There is also quantitative agreement between the magnetic field strength observed and that calculated by using an EMF produced by Coriolis forces and given in Drobishevskii, 1968. [Condensed. [Ed.).] [Drobishevskii, V. G.: 1968, Astrofiz. 4, 537 (under translation for Astrophys.).]

\section{Various Questions to Boyarchuk}

Verschuur: I have made $21-\mathrm{cm}$ line observations in directions of four recurrent novae. I have found that a peculiar cloud structure exists in the direction of two of these novae (for the other two the data are not yet reduced). To interpret these data, I need answers to the following questions: How long do recurrent novae operate? What is the age of a recurrent nova? What is the total mass ejected during the lifetime? Can we expect to see this mass directly? Can we observe this mass in a neutral state if it is ejected as hot matter and then cools at great distances from the star? Can we see the effect that the nova had on the surrounding medium? Can the energy ejected by the nova effectively be converted to kinetic energy of the surrounding medium? I do not think answers to all these questions exist, but I would like to hear a discussion of them, either by Boyarchuk or by Pottasch.

Boyarchuk: I am afraid that we have no good answers to all these questions, but I will discuss some of them. We know eight recurrent novae and only two have been known for a long time - T Cor Bor and RS Oph. T Cor Bor was recorded at the end 
of the last century, therefore we can say only that the activity of recurrent novae has been continuous for 100 years at least. Now about the mass. It is very difficult to give an accurate value for the mass per outburst for the following reasons. Suppose a recurrent nova has consecutive outbursts separated by 30 years. During the outburst, we see many emission lines, but we cannot be sure where the emission lines are formed. They may be formed in an envelope consisting of material ejected during the ongoing outburst, or in an envelope consisting of material from all previous outbursts. Because material is ejected with high speed, a shock wave can be generated exciting all the material collected in the envelope. Therefore, we do not know whether the figure of $10^{-7} M_{\odot}$ in the shell refers to one outburst or to many. Another difficulty is that the velocity of expansion is large for recurrent novae (perhaps $4000 \mathrm{~km}$ $\mathrm{sec}^{-1}$ ) and therefore the material disappears very quickly. I do not know enough about the other questions.

Pottasch: I could discuss one question, perhaps. There is evidence in RS Oph that during the last outburst there was already a substantial amount of material surrounding the star (Pottasch, 1968). How it got there is an open question. It might have arrived there between outbursts, or it might have been the product of a previous outburst. The evidence for this mass is the existence of very narrow absorption and emission lines that are observed during the outburst and the fact that the material in the outburst and the shell seems to be decelerated. It looks as if there is a mass out there in approximate hydrostatic equilibrium that extends quite a distance from the star. Presumably, between outbursts this cools and might be neutral and observable. (Pottasch, S. R.: 1968, Bull. Astron. Inst. Netherl. 19, 227.)

Thomas: It seems to me that Pottasch is saying that material is being pumped out which does not go far enough to escape.

Pottasch: It looks as if there were a lot of material surrounding the nova. The evidence for it is the $\mathrm{H} \alpha$ line you observe. Superimposed on the usual, broad P Cygtype profile you see a very narrow $\mathrm{P}$ Cyg-type profile (with a width of the order of $10 \mathrm{~km} \mathrm{sec}^{-1}$ ). When the outburst takes place, it sweeps up the material that surrounds the star, and this narrow line decreases in intensity with time, since less material remains. At the same time the absorption velocity of the main profile shows that the material involved in the outburst is being decelerated. The deceleration probably occurs because of the effect of the sweeping up of the existing medium far out. This all leads to a consistent picture with rough hydrostatic equilibrium in a large amount of material surrounding the nova before its outburst. It may be moving with small speeds, of the order of 5 or $10 \mathrm{~km} \mathrm{sec}^{-1}$.

Thomas: Is it moving away or back?

Pottasch: It is moving away from the nova.

Habing: Dr. Pottasch, could you give an angular size for this shell?

Pottasch: You can give it an absolute size of about $10^{15} \mathrm{~cm}$ before the density gets so low that it is no longer observable.

Habing: Such sizes exclude observation of associated $21-\mathrm{cm}$ emission-line profiles. 
Shklovskii: Can a regular nova be in fact a recurrent nova with a very long period between outbursts, say of the order of $2000 \mathrm{yr}$ ?

Boyarchuk: There are differences. In the first place, a regular nova consists of a binary system in which the two components are both dwarf stars, and the rotation period is only a few hours. But the only recurrent nova that has been studied closely, T Cor Bor, presents a different picture. Again, it is a binary system; but one component is a red giant and the other component is a hot, small star. The rotation period is something like 250 days. In the second place there are spectroscopic differences. The recurrent novae have a higher degree of excitation (coronal lines from Fex, etc.). In regular novae we never have such strong coronal lines. Third, the mass of the envelope of regular novae is about $10^{-4} M_{\odot}$ but for recurrent novae, we have $10^{-7} M_{\odot}$ in the envelope.

Gordon: Is there some difference in the space distribution between the ordinary and the recurrent novae?

Boyarchuk: We do not know the space distribution of the recurrent novae because only eight recurrent novae are known.

Van de Hulst: Dr. Boyarchuk, I got the impression that each mass loss determination was based on several spectrograms taken at one time. Did you repeat the analysis subsequently with a number of spectra taken at several different times after the nova outburst, and were the consecutive results consistent?

Boyarchuk: I studied nova envelopes only a long time after the outburst and so it does not matter if you investigate today or next year, because the ejected material is the same and the excitation conditions do not change strongly. But during the outburst, or just after the outburst, of course, these conditions change rapidly.

Shklovskii: Dr. Boyarchuk, if you determine the mass of emission nebulae, the result will be strongly influenced by inhomogeneities in the density. But if you use absorption lines, the inhomogeneities will not be so important. So the question is: Is there some difference between the mass determined from absorption lines and that from emission lines?

Boyarchuk: There is no single object, for which we have reliable mass determinations in the two ways you described. In the case of nebulae and novae where we have large envelopes, we have no absorption lines. But for $\mathrm{T}$ Tau stars, where we use absorption lines for mass-loss determinations, we do not observe an envelope. Only for the symbiotic stars is there in principle the possibility for such a comparison. But here the comparison is not very meaningful, because there are many uncertainties, such as the frequency of large flares, how long they last, and the amount of material ejected per flare. In the one case available in the literature both methods give the same order of magnitude for the mass-loss rate.

Shklovskii: But if you have the same results from the two cases then it follows that there are no inhomogeneities in the envelope.

Thomas: I have a simple pictorial comment on your question. In the literature one often finds the optical thickness of flares, hence the line-of-sight mass in a flare, determined from the equation 


$$
I_{F}=I_{p} e^{-\tau}+\int_{0}^{\tau} B e^{-\tau} \mathrm{d} \tau,
$$

where $I_{F}$ is the intensity in the flare. To determine $\tau$ one assumes that $I_{p}$, the intensity at the base of the flare, can be obtained from observations of non-flare regions; and by comparing $I_{F}$ and $I_{p}$, one gets $\tau$ by making some assumption on $B$ in the flare. The problem is that this can lead to orders of magnitude error in the value of $\tau$, hence in the value of the mass of the flare. The error lies in the fact that $I_{p}$ depends very much on whether or not there is overlying material. This procedure is therefore completely invalid. One requires an analysis that takes into account the influence of the material in the flare upon the value of $I_{p}$. This is the same kind of problem, intrinsically, as that of determining the amount of material in the chromosphere, or in the mass of a shell. As an example, early estimates gave $\tau \approx 1$ for the chromosphere in $\mathrm{H} \alpha$; modern determinations give $\tau \approx 100$. The same kind of error can enter the determination in the cited cases of flares and stellar shells. The absorption part of the P Cyg profile would be treated as above. The emission part would be treated differently. Thus, depending upon whether or not we use a correct theory for each, we can find differences in results which can reflect an error in the theory of analysis rather than in the physical situation or the presence of inhomogeneities.

Gershberg: I should like to say a few words about UV Ceti flare stars and their possible relevance for the interstellar medium. This type is the most numerous of all eruptive stars. We have one UV Ceti-type star per $12 \mathrm{pc}^{3}$ and out of 45 stars nearer than $5 \mathrm{pc}, 8$ belong to this type. In quiet periods the stars show strong $\mathrm{H}$ and $\mathrm{Ca}$ emission lines. A preliminary analysis indicates that the stars have important chromospheres and that the number of radiating $\mathrm{H}$-atoms under $1 \mathrm{~cm}^{2}$ of their photosphere is one or two orders of magnitude higher than in the Sun. The radii are in the range of 0.1 to $0.3 R_{\odot}$ and the masses in the range of 0.1 to $0.3 M_{\odot}$. Because of the presence of an important chromosphere and the similarity of the gravitational potential $(g \propto M / R)$ for the Sun and the stars we expect a big corona and stellar wind. Kahn (1969) arrived at similar conclusions from the analysis of the radio flare of YZ CMi. Evaluation of the soft X-ray emission from UV Ceti-type stars gives a few per cent of the total observed soft X-ray flux. But if this flux is mainly from an extragalactic origin, the stellar source will be dominant in the direction of highly obscured regions like the Ophiuchus and the Taurus areas. The soft X-ray flux from UV Ceti-type stars is not yet observed during the flare, however. Two years ago we compared simultaneous photoelectric observations of several flare stars and observations of radio emission at 13 and $26 \mathrm{MHz}$. During stellar flares no radio emission was observed and an upper limit of $F_{x} / F_{\text {opt }}<10^{5}$ was obtained. This observed upper limit is very much larger than the expected ratio. But one has to keep in mind that in stellar flares the ratio $F_{\text {radio }}$ $F_{\text {opt }}$ is sometimes 100 times higher than in solar flares. (Kahn, F. D.: 1969, Nature 222, 1130.) 